\title{
In-situ bioconjugation in stationary media and in liquid flow by femtosecond laser ablation
}

\author{
Csaba László Sajti • Svea Petersen • \\ Ana Menéndez-Manjón • Stephan Barcikowski
}

Received: 9 November 2009 / Accepted: 10 May 2010 / Published online: 16 June 2010

(C) The Author(s) 2010. This article is published with open access at Springerlink.com

\begin{abstract}
In-situ functionalization of gold nanoparticles with fluorophore-tagged oligonucleotides is studied by comparing femtosecond laser ablation in stationary liquid and in biomolecule flow. Femtosecond laser pulses induce significant degradation to sensitive biomolecules when ablating gold in a stationary solution of oligonucleotides. Contrary, in-situ conjugation of nanoparticles in biomolecule flow considerably reduces the degree of degradation studied by gel electrophoresis and UV-Vis spectrometry. Ablating gold with $100 \mu \mathrm{J}$ femtosecond laser pulses DNA sequence does not degrade, while the degree of fluorophore tag degradation was $84 \%$ in stationary solution compared to $5 \%$ for $1 \mathrm{~mL} / \mathrm{min}$ liquid flow. It is concluded that femtosecond laser-induced degradation of biomolecules is triggered by absorption of nanoparticle conjugates suspended in the colloid and not by ablation of the target. Quenching of nanoparticle size appears from $0.5 \mu \mathrm{M}$ biomolecule concentration for $0.3 \mu \mathrm{g} / \mathrm{s}$ nanoparticle productivity indicating the successful surface functionalization. Finally, increasing the liquid flow rate from stationary to $450 \mathrm{~mL} / \mathrm{min}$ enhances nanoparticle productivity from $0.2 \mu \mathrm{g} / \mathrm{s}$ to $1.5 \mu \mathrm{g} / \mathrm{s}$, as increasing liquid flow allows removal of light absorbing nanoparticles from the ablation zone, avoiding attenuation of subsequent laser photons.
\end{abstract}

C.L. Sajti · S. Petersen · A. Menéndez-Manjón ·

S. Barcikowski ( $\square)$

Laser Zentrum Hannover e.V., Hollerithallee 8, 30419 Hannover,

Germany

e-mail: s.barcikowski@lzh.de

Fax: +49-511-2788100

\section{Introduction}

Nanoparticles, especially plasmonic materials such as gold nanoparticles characterized by highly enhanced resonant absorption and scattering properties, are particularly useful in cell targeted drug delivery [1], high resolution bioimaging [2,3], biomedical diagnostics and therapeutics [4] when conjugated with functional molecules (e.g. oligonucleotides). During the last decade, a variety of methods for gold nanoparticle preparation have been established. Among these, the standard chemical synthesis is based on the reduction of $\mathrm{Au}(\mathrm{III})$ in presence of reducing agents such as citrate [5] or sodium borohydride [6] and stabilizing ligands, while bioconjugation was described by directly adding conjugative agents during the reduction process. These chemical techniques might have a critical drawback involving the use of chemical precursors and reducing agents requiring a purification procedure where residual ligands limit subsequent conjugation.

Pulsed laser ablation in liquids (PLAL) is a recently emerged alternative single step approach enabling sizecontrolled generation of stable nanoparticle colloids [7-10] with outstanding purity and novel surface chemistry not reproducible by any other conventional manufacturing route. Recently, Sylvestre et al. revealed the presence of the oxidation states $\mathrm{Au}^{+}$and $\mathrm{Au}^{3+}$ besides the metallic $\mathrm{Au}^{0}$ due to partial oxidation of the nanoparticles in aqueous media [9]. Owing to this partial oxidation, laser-generated gold nanoparticles act as electron acceptors; hence, they are easily coordinated by molecules bearing electron donor moieties as thiol, amine or carboxyl groups added to ablation media prior (in-situ conjugation) or after (ex-situ conjugation) the laser process. However, we recently published that in-situ conjugation leads to about four times higher conjugation efficiency than ex-situ functionalization reaching $29 \mathrm{pmol} \mathrm{cm}^{-2}$ [11]. 
Although ultrashort pulsed laser presents a promising tool for in-situ bioconjugation, due to minimal thermal impact to ablated material and surrounding media when ablating with laser fluences close to the ablation threshold [12], it has been already shown to induce similar residual thermal effects as nanosecond lasers in the high fluence regime [13]. Furthermore, femtosecond pulses has been already found to melt and photothermally reshape ablated gold nanoparticles in water [14] and also to induce certain degradation to gold conjugates, however the degradation mechanism still remains unclear [15].

In this paper, we describe the in-situ conjugation of gold nanoparticles with oligonucleotides by ultrashort pulsed laser ablation in stationary liquid and in a controlled biomolecule flow. Degree of biomolecule degradation was quantitatively investigated and the possible mechanisms of femtosecond laser-induced DNA degradation are discussed and show the trade-off between quality and nanoparticle production rate.

\section{Experimental details}

\subsection{Nanoparticle generation method}

Ultrashort PLAL was carried out using a commercial femtosecond laser system (Spitfire Pro, Spectra-Physics) delivering $120 \mathrm{fs}$ laser pulses with $5 \mathrm{kHz}$ repetition rate at a central wavelength of $800 \mathrm{~nm}$. Laser irradiation was focused onto the surface of a $100 \mu \mathrm{m}$ thick gold foil (Goodfellow $\mathrm{GmbH}$ ) merged into $4 \mathrm{~mm}$ of liquid layer. During nanoparticle generation the ablation chamber was continuously moved with a three axis positioning system (3D Microcac) providing constant lateral speed of $1 \mathrm{~mm} \mathrm{~s}^{-1}$ in a spiral ablation pattern. Two experimental concepts were utilized, performing laser ablation in stationary liquid and in biomolecule flow, presented schematically in Fig. 1. Ablation in a stationary liquid was performed in a glass vessel filled with $500 \mu \mathrm{L}$ of aqueous solution $\left(\mathrm{H}_{2} \mathrm{O}:>18 \mathrm{M} \Omega \mathrm{cm}\right.$, Millipore $)$ of thiolated single stranded oligonucleotides ( $\mathrm{ssO}$ ). In a second experimental arrangement gold nanoparticle ssO conjugates were synthesized in a controlled oligonucleotide flow generated by a digital syringe pump (NE-300, New Era) using a self-constructed flow chamber presented in Fig. 1 b.

\subsection{Characterization techniques}

Degree of degradation was investigated by gel electrophoresis and by UV-Vis absorption in the spectral region of 220-800 nm (Shimadzu 1650), of gold nanoparticles functionalized by a thiolated $\mathrm{ssO}$ sequence (5'cta-cct-gca-ctgtaa-gca-ctt-tg-3', Mod. 5':C6-Disulfide, Biospring GmbH) and by $\mathrm{ssO}$ sequence tagged by Cy 5 fluorophore (Cy5-ssO 5' agc-aca-tct-cgg-tcc-ctg-3', Mod. 3' C6-Disulfide, Mod. 5' Cy5, Purimex DNA/RNA Oligonucleotides), respectively. The ablation media after nanoparticle generation were treated with $5 \mathrm{mM}$ aqueous dithiothreitol-solution (DTT) to displace surface bound ssO via an exchange reaction and induce nanoparticle aggregation as consequence. This method guarantees that both conjugated and unconjugated ssO are analyzed quantitatively. The ssO containing ablation media were loaded on a $20 \%$ denaturing polyacrylamide gel, run for 3 hours at $300 \mathrm{~V}$ and silver stained. To quantify the integrity of ssO after irradiation, we compared the intensity of intact $\mathrm{ssO}$ band with the intensity of the $\mathrm{ssO}$ band prior to nanoparticle generation. To visualize the bands, gels were stained and developed using FastSilver (G Biosciences, USA). Scans of resulting stained gels were analyzed with Image $\mathbf{J}$ software. Biomolecule degradation was also quantified by the Cy 5 absorption band at $648 \mathrm{~nm}$ by UV-Vis spectrometry. For this purpose, the ablated colloids were first treated by DTT, and in a next step gold nanoparticles were forced to agglomerate by $200 \mathrm{mM}$ aqueous $\mathrm{NaCl}$ solution. After 10 minutes of centrifugation at $5000 \mathrm{rpm}$ the absorption spectrum of $\mathrm{Cy} 5$-ssO containing supernatant were analyzed. Size measurements were performed by dynamic light scattering (DLS, Zetasizer ZS Malvern Instruments) and by transmission electron microscopy (TEM, EM 10C Zeiss), while the nanoparticle production rate was determined by
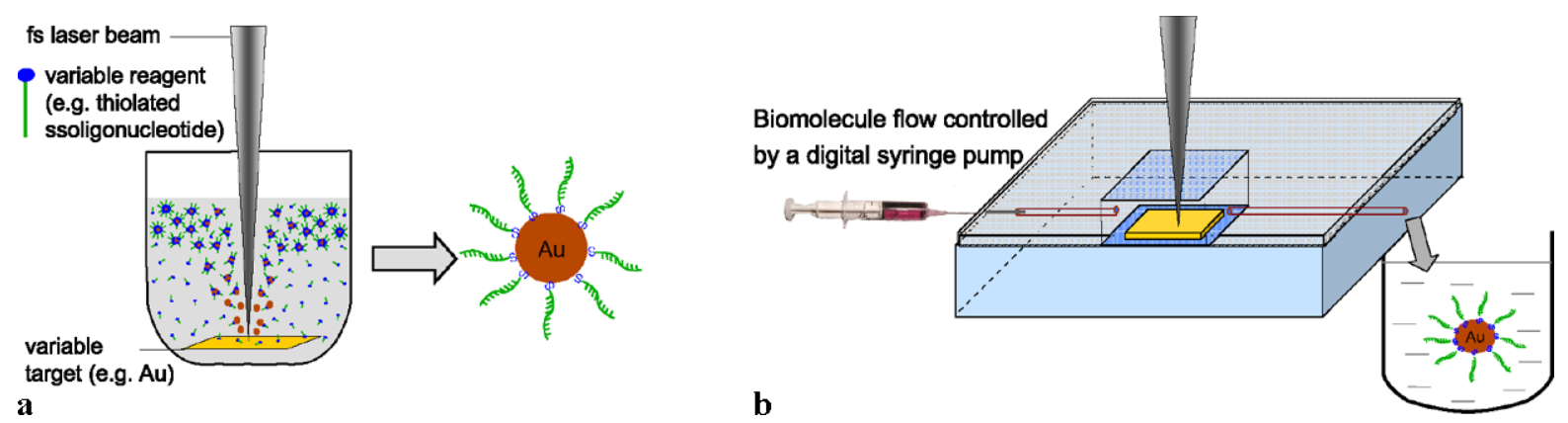

Fig. 1 Schematic illustration of the in-situ bioconjugation in stationary liquid (a) and in biomolecule flow (b) by femtosecond laser ablation 
differential weighting of the target by a precision microbalance (Sartorius M3P) via the estimation that no material was attached on the process chamber wall.

\section{Results and discussion}

During laser ablation, the ejected material is in a chemically extremely active state of growth and it is well known that nanoparticle size can be significantly influenced by the amount of conjugative agents in solution. Figure 2a shows the Feret and hydrodynamic diameters of nanoparticle bioconjugates in function of the $\mathrm{ssO}$ concentration obtained by $100 \mu \mathrm{J}$ of pulse energy in stationary liquid. Reciprocal dependency of primary nanoparticle size on the concentration of $\mathrm{ssO}$ was clearly revealed. That is in good agreement with data obtained in a previous study for conjugation with Dodecanthiol in hexane [16]. The significant difference between nanoparticle size by TEM and DLS is due to the fact that hydrodynamic size of nanoparticles is strongly affected by the presence of DNA molecules and by solvation of conjugates. It seems that a critical concentration of $\mathrm{ssO}$ is required to achieve a significant growth quenching. As the concentration falls below $0.5 \mu \mathrm{M}$, agglomerates are detected which could also be visualized by the quotient of the plasmon resonance spectrum at 800 and $380 \mathrm{~nm}$. Ablating with higher ssO concentrations no further size decrease was observed and a plateau in both Feret and hydrodynamic particle size appeared. TEM micrographs also confirmed this tendency (Fig. 2b).

As known from literature, ablation efficiency and nanoparticle yield increase with pulse energy, although at the same time, heat impact to material and the risk of molecule degradation also increases [17]. Process window of negligible degradation of biomolecules with highest ablation rate have to be identified. We thus examined nanoparticle production rate at focal position with different pulse energies in stationary $3 \mu \mathrm{M}$ ssO solution and studied oligonucleotide degradation by gel electrophoresis in parallel, shown in Figs. 3a and 3b, respectively. Nanoparticle productivity increased with pulse energy, reaching $1 \mu \mathrm{g} \mathrm{s}^{-1}$ with $200 \mu \mathrm{J}$ focused laser pulses corresponding to about $8 \times 10^{11}$ conjugate $\mathrm{s}^{-1}$ with average size of $5 \mathrm{~nm}$. This yield is higher than the one obtained by femtosecond PLAL of gold in pure water being only $0.2 \mu \mathrm{g} \mathrm{s}^{-1}$ or about $6 \times 10^{9}$ nanoparticle $\mathrm{s}^{-1}$ considering an average particle diameter of $15 \mathrm{~nm}$. As published earlier, ablating in water, gold nanoparticles tend to redeposit on the target's surface after ablation, whilst during in-situ functionalization they remain in the solution, enhancing production rate of colloidal particles [16]. Besides enhanced ablation rate, degree of oligonucleotide disintegration also increases with pulse energy, presented in Fig. $3 b$. After one minute of ablation, no degradation of $\mathrm{ssO}$ was observed up to $100 \mu \mathrm{J}$ focused pulse energy, followed by a rapid increase in photo/thermal-induced cleavage of DNA sequences. Ablation with $200 \mu \mathrm{J}$ femtosecond laser pulses causes significant degradation of about $22 \%$. As already published, activation energy of heat-induced DNA depurination followed by cleavage of nearby phosphor-diesters is $127 \mathrm{~kJ} \mathrm{~mol}^{-1}[17,18]$. We roughly estimated the molar energy density in the vicinity of the focus position to be $6.8 \mathrm{GJ} \mathrm{cm}^{-2} \mathrm{~mol}^{-1}$ considering $200 \mu \mathrm{J}$ pulse energy, $50 \mu \mathrm{m}$ focal diameter and $3 \mu \mathrm{M}$ concentration in a total volume of $500 \mu \mathrm{L}$, neglecting the nonlinear absorption cross-section of
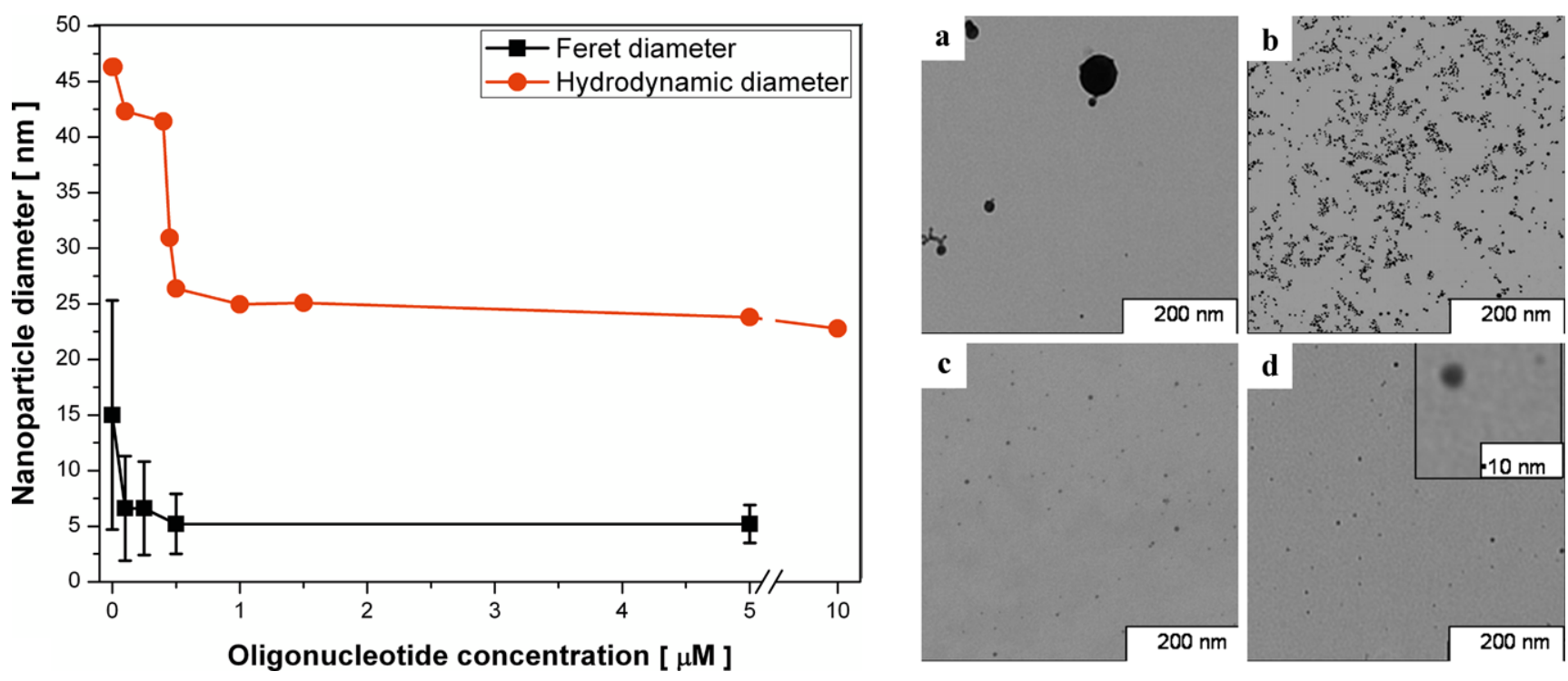

Fig. 2 Influence of oligonucleotide concentration on the hydrodynamic and Feret diameters of laser-generated bioconjugates (left) and TEM images of pure gold nanoparticles (a) and bioconjugates synthesized by $0.1 \mu \mathrm{M}(\mathbf{b}), 0.5 \mu \mathrm{M}(\mathbf{c})$ and by $5 \mu \mathrm{M}(\mathbf{d})$ biomolecule concentration 

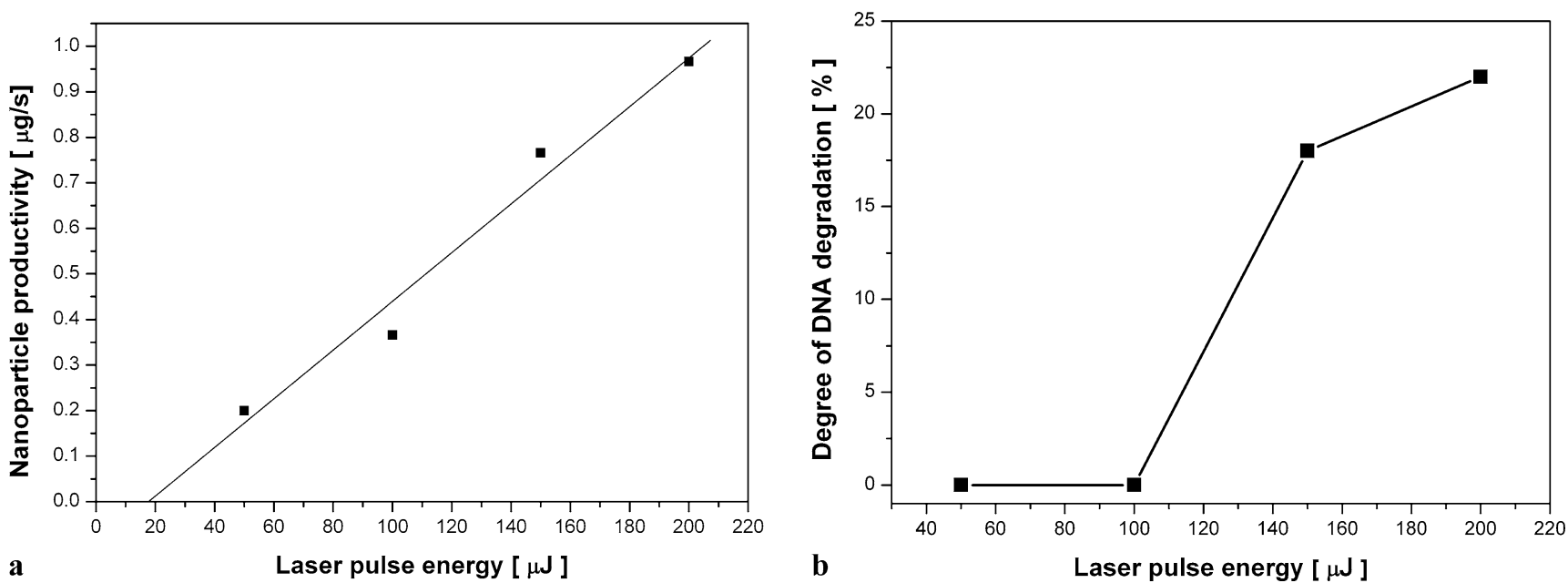

Fig. 3 Gold nanoparticle productivity (a) and corresponding degree of oligonucleotide degradation (b) as a function of the focused laser pulse energy determined by gel electrophoresis using $3 \mu \mathrm{M}$ DNA concentration
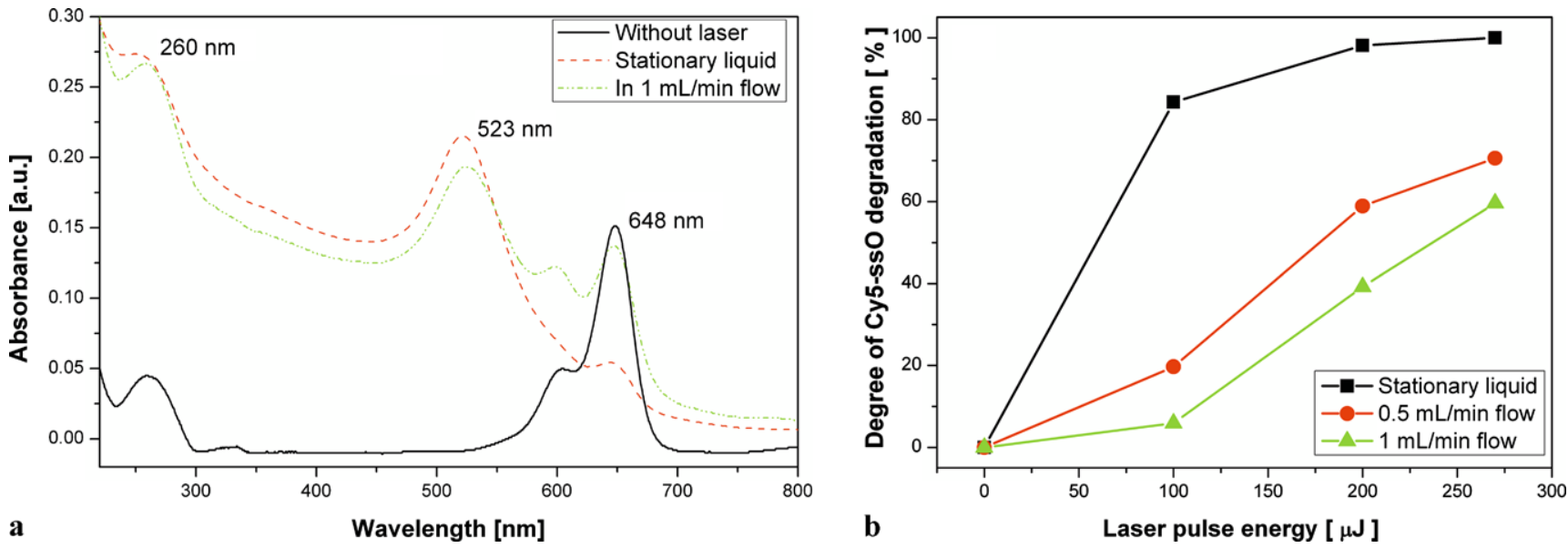

Fig. 4 Absorption spectrum of nanoparticle bioconjugates (DNA $260 \mathrm{~nm}$, gold nanoparticle $\sim 523 \mathrm{~nm}$, fluorophore tag $\sim 648 \mathrm{~nm}$ ) ablated with $100 \mu \mathrm{J}$ pulse energy in stationary liquid and in biomolecule

flow (a). Degree of Cy5-labeled oligonucleotide degradation at $648 \mathrm{~nm}$ in function of laser pulse energy (b)

such DNA sequence for $800 \mathrm{~nm}$ as it is not yet published. Due to the fact that this high amount of energy is deposited within the time scale faster than the electron phonon coupling, being about 4.7 ps for gold [19], fabricating nanoparticles with femtosecond laser ablation close to the ablation threshold remains a cold process, and laser energy seems to be deposited mainly at the gold target. In order to investigate whether the laser ablation itself, or the presence of nanoparticles trigger biomolecule degradation during femtosecond PLAL, we ablated gold in the presence of $1.5 \mu \mathrm{M}$ Cy5-tagged ssO with various pulse energies in stationary solution and in biomolecule flow and studied the main absorption peak of Cy5 at $648 \mathrm{~nm}$. Figure 4a clearly demonstrates the difference between reference biomolecule spectrum and absorption spectra obtained by the two experimental concepts using $100 \mu \mathrm{J}$ pulse energy. It was clearly re- vealed that femtosecond laser ablation in stationary Cy5$\mathrm{ssO}$ media induces drastic degeneration to the fluorophore tag, while ablation in a biomolecule flow with same laser parameters minimizes degradation. In the spectra of bioconjugates, besides the principal peak and by-peak of $\mathrm{Cy} 5$ at $648 \mathrm{~nm}$ and $604 \mathrm{~nm}$ respectively one observes a second band at $260 \mathrm{~nm}$. This transition results from the electronical shifts in purines and pyrimidines contained in the oligonucleotide. Figure $4 \mathrm{~b}$ depicts quantitatively the degree of Cy5labeled oligonucleotide degradation at $648 \mathrm{~nm}$ in function of laser pulse energy and liquid flow rate. As expected, degree of degradation reduces considerably with higher flow rates. Since only the total residence time of ablated species in the ablation zone is varied by the liquid flow rate, we assume that biomolecule-fluorophore disintegration by femtosecond laser irradiation is mainly induced and amplified by ab- 


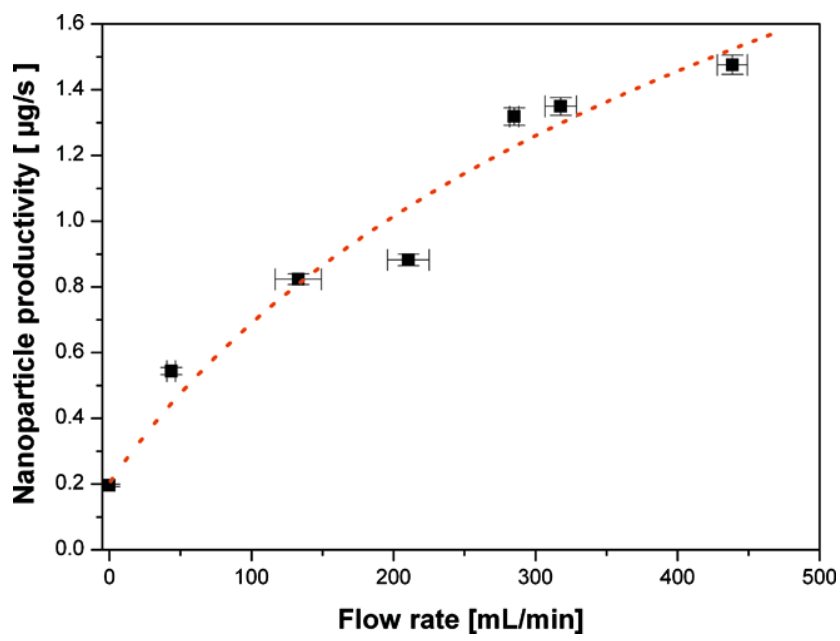

Fig. 5 Gold nanoparticle productivity in water in function of liquid flow velocity using $100 \mu \mathrm{J}$ focused laser pulse energy

sorption and scattering of subsequent laser beam on previously ablated, suspended nanoparticles/nanoparticle conjugates in the colloidal solution and not triggered directly by the ablation itself. Applying $100 \mu \mathrm{J}$ focused laser pulses for 1 minute, degree of Cy5-ssO degradation was measured to be $84 \%$ for gold ablation in stationary oligonucleotide solution, $20 \%$ using $0.5 \mathrm{~mL} / \mathrm{min}$ biomolecule flow and only $5 \%$ using $1 \mathrm{~mL} / \mathrm{min}$ flow rate. Using $1 \mathrm{~mL} / \mathrm{min}$ of liquid flow, the ablated species are estimated to leave the vicinity of the focal point in the time scale of about a few microseconds, hence faster than the time constant determined by the laser repetition rate $(200 \mu \mathrm{s})$. We thus assume that not ideal laminar liquid flow results in convection of nanoconjugates into the entire volume of the ablation chamber (about $100 \mu \mathrm{L}$ ), and they might be further reirradiated with subsequent laser pulses, resulting in a small degree of biomolecule degradation.

Besides the prevention of photo-degradation, increasing liquid flow rate was found to lead to an important rise of nanoparticle productivity using $100 \mu \mathrm{J}$, presented in Fig. 5 . For this purpose, we utilized a high power peristaltic pump (PD 5001, Heidolph Instruments). Raising liquid flow speed from stationary to $450 \mathrm{~mL} / \mathrm{min}$ resulted in a significant increase of nanoparticle production rate from $0.2 \mu \mathrm{g} / \mathrm{s}$ to $1.5 \mu \mathrm{g} / \mathrm{s}$, respectively, while we did not observe significant variation in nanoparticle size and thus assumed that fragmentation might take place for nanoparticles under longer post-irradiation times. Ablation rate increased about logarithmically with the outflow velocity and it seems to begin to saturate at $450 \mathrm{~mL} / \mathrm{min}$. Without liquid drain/flow, the ablated nanoparticles disperse slowly into the entire liquid. Thus after each ablation sequence a dense particle cloud is ejected characterized by a relatively long residence time in front of the target leading to significant absorption of the subsequent laser pulse.

\section{Conclusion}

Femtosecond laser ablation of gold in aqueous solution of thiolated oligonucleotides was established as a powerful single step approach to conjugate oligonucleotide sequences to gold nanoparticles in situ. Quenching of nanoparticle size appeared from $0.5 \mu \mathrm{M}$ biomolecule concentration for $0.3 \mu \mathrm{g} / \mathrm{s}$ nanoparticle productivity clearly indicating the successful surface functionalization. Quantitative investigation of biomolecule signals by gel electrophoresis and UV-Vis spectrometry of Cy5-tagged oligonucleotides revealed that generation of bioconjugates in stationary liquid is drastically limited by laser pulse energy as significant degradation occurs during ablation. Functionalization of nanoparticle surface in a biomolecule flow with equal laser parameters radically reduces the degree of degradation even for high laser fluences. Ablating gold with $100 \mu \mathrm{J}$ focused laser pulse energy, integrity of oligonucleotides was $16 \%$ for stationary and $95 \%$ for $1 \mathrm{~mL} / \mathrm{min}$ oligonucleotide flow. We assume that femtosecond laser-induced photo-degradation of biomolecules is mainly caused by absorption/scattering of subsequent laser beam by colloidal nanoparticle conjugates. Increasing liquid flow rate from stationary to $450 \mathrm{~mL} / \mathrm{min}$ allowed the prompt removal of nanoparticles from the ablation zone, resulting in a significant increase of nanoparticle production rate from $0.2 \mu \mathrm{g} / \mathrm{s}$ to $1.5 \mu \mathrm{g} / \mathrm{s}$, during femtosecond laser ablation in water with $100 \mu \mathrm{J}$ laser pulses.

Acknowledgements This work was supported by funding from the Deutsche Forschungsgemeinschaft (DFG, German Research Foundation) for the Cluster of Excellence REBIRTH (From Regenerative Biology to Reconstructive Therapy) and by the research project CH 179/9-1.

Open Access This article is distributed under the terms of the Creative Commons Attribution Noncommercial License which permits any noncommercial use, distribution, and reproduction in any medium, provided the original author(s) and source are credited.

\section{References}

1. S. Aryal, J.J. Grailer, S. Pilla, D.A. Steeben, S. Gong, J. Mater. Chem. 19, 7879 (2009)

2. K.T. Yong, M.T. Swihart, H. Ding, P.N. Prasad, Plasmonics 4, 79 (2009)

3. M. Bruchez, M. Moronne, P. Gin, S. Weiss, A.P. Alivisatos, Science 281, 2013 (1998)

4. I. Brigger, C. Dubernet, P. Couvreur, Adv. Drug Deliv. Rev. 54, 631 (2002)

5. J. Turkevich, P.C. Stevenson, J. Hillier, Discuss. Faraday Soc. 11, 55 (1951)

6. M. Brust, M. Walker, D. Bethell, D.J. Schiffrin, R. Whyman, J. Chem. Soc., Chem. Commun. 801 (1994)

7. F. Mafunè, J. Kohno, Y. Takeda, T. Kondow, J. Phys. Chem. B 104, 9111 (2000)

8. J.P. Sylvestre, A.V. Kabashin, E. Sacher, M. Meunier, Appl. Phys. A 80, 753 (2005) 
9. J.P. Sylvestre, S. Poulin, A.V. Kabashin, E. Sacher, M. Meunier, J.H.T. Luong, J. Phys. Chem. B 108, 16864 (2004)

10. S. Barcikowski, F. Devesa, K. Moldenhauer, J. Nanopart. Res. 11, 1883 (2009)

11. S. Petersen, S. Barcikowski, J. Phys. Chem. C 113, 19830 (2009)

12. B.N. Chichkov, C. Momma, S. Nolte, F. von Alvensleben, A. Tünnermann, Appl. Phys. A 63, 109 (1996)

13. A.Y. Vorobyev, C. Guo, J. Phys., Conf. Ser. 59, 418 (2007)

14. S. Link, C. Burda, B. Nikoobakht, M.A. El-Sayed, J. Phys. Chem. B 104, 6152 (2000)
15. S. Besner, A.V. Kabashin, F.M. Winnik, M. Meunier, J. Phys. Chem. C 113, 9526 (2009)

16. S. Petersen, J. Jakobi, A. Hörtinger, S. Barcikowski, J. Laser Micro Nanoeng, 4, 71 (2009)

17. S. Petersen, S. Barcikowski, Adv. Funct. Mater. 19, 1 (2009)

18. L. Zhang, Q. Wu, J. Biosci. Bioeng. 30, 599 (2005)

19. M. Ligges, I. Rajkovic, P.X. Zhou, O. Posth, C. Hassel, G. Dumpich, D. von der Linde, Appl. Phys. Lett. 94, 101910 (2009) 